November - 2005

\title{
Remote Access to Instrumental Analysis for Distance Education in Science
}

\author{
Dietmar Kennepohl, Athabasca University - Canada’s Open University \\ Jit Baran, Northern Alberta Institute of Technology, Canada \\ Martin Connors, Athabasca University - Canada’s Open University \\ Kieron Quigley, Northern Alberta Institute of Technology, Canada \\ Ron Currie, Northern Alberta Institute of Technology, Canada
}

\begin{abstract}
Remote access to experiments offers distance educators another tool to integrate a strong laboratory component within a science course. Since virtually all modern chemical instrumental analysis in industry now use devices operated by a computer interface, remote control of instrumentation is not only relatively facile, it enhances students' opportunity to learn the subject matter and be exposed to "real world" contents. Northern Alberta Institute of Technology (NAIT) and Athabasca University are developing teaching laboratories based on the control of analytical instruments in real-time via an Internet connection. Students perform real-time analysis using equipment, methods, and skills that are common to modern analytical laboratories (or sophisticated teaching laboratories). Students obtain real results using real substances to arrive at real conclusions, just as they would if they were in a physical laboratory with the equipment; this approach allows students to access to conduct instrumental science experiments, thus providing them with an advantageous route to upgrade their laboratory skills while learning at a distance.
\end{abstract}

\section{Introduction}

For most of us the term "remote control" conjures up images of kids playing with radio controlled model cars and airplanes or some sort of rampaging science fiction robot manipulated by the villain in a story. Yet remote control is well incorporated into our growing technological world. We have automatic car starters on our key chains, police bomb squads regularly make use of robots, un-manned reconnaissance drones are used by the military, and most of us use a remote control to change television channels.

The concept of using remote control in the sciences is not new either. Scientists often exploit the advantages of remote access when the experiment they wish to conduct is physically inaccessible by virtue of location or danger. For example, safety demands that all nuclear fission reactors are operated remotely and the location of the orbiting Hubble space telescope makes remote control a 
necessity. One stunning high profile example of remote control is the landing of the NASA twin exploration rovers Spirit and Opportunity on Mars in January 2004. These rovers are now physically and chemically exploring that planet while being controlled from Earth.

A strong laboratory component is at the heart of many science courses. It is also one of the more challenging components to deliver effectively at a distance (Kennepohl and Last, 1997). There is no one correct solution or technology and often an assortment of methods are used in concert to overcome these challenges. The methodology and success of varying approaches to delivering science laboratories at Athabasca University has been discussed elsewhere in some detail (Holmberg and Bakshi, 1992; Kennepohl and Last, 2000; Connors, 2004). In each instance, however, the intent is to offer students a laboratory experience equivalent to, but not necessarily identical to, that they would encounter in a more traditional face-to-face setting.

With the availability of the World Wide Web, both campus and distance-based educators in disciplines like biology, chemistry, physics, and engineering, which traditionally have a strong laboratory component, have been exploring the integration of experiments into their face-to-face and online courses. In many instances, the online laboratory components are simulated and offer the "so-called" virtual laboratory experience (Kennepohl, 2001). A few groups, however, have directed their efforts towards allowing students remote access to real experiments. For example, an optical experiment at Stanford University in the United States is described under the Cyberlab project (Hesselink, Rizal, and Bjornson, 2000). There is also the PEARL project (Practical Experimentation by Accessible Remote Learning) which is a consortium of European Union (EU) institutions developing remote experiments in spectrometry, cell biology, manufacturing engineering, and electronic engineering (Cooper, et al., 2000). Another EU consortium called Network for Education - Chemistry uses mostly interactive simulations, but is also exploring online remote process control by way of a residence time distribution experiment (Zurn, Paasch, Thiele, and Salzer, 2003). Our own research has primarily focused on controlling sophisticated analytical instruments in chemistry (Baran, Currie, and Kennepohl, 2004).

Most modern analytical instruments are intimately controlled by computer, thus making access by remote control possible. Several others have already reported using the commercially available LabVIEW software system for controlling instrumentation and acquiring data in an in-class teaching environment (Drew, 1996; Gostowski, 1996; Haines, 1998; Spanoghe, Cocquyt, and Van der Meeren, 2001). In a recent pilot study, which allowed chemistry students to control analytical instruments in real-time, we demonstrated that a client-server application can be achieved from a remote location over the Internet, with an acceptable level of security, using $P C$ Duo software (Baran et al., 2004). Although the initial pilot study has shown the viability of this concept, the present work describes adapting this technology to a teaching environment that allows students both facile access to instrumental chemistry experiments and an advantageous route to upgrade their laboratory skills at a distance. Bernard and coworkers recently presented evidence through a comprehensive meta-analysis of empirical literature that distance education was generally comparable with classroom instruction on a number of variables including retention and learning outcomes (Bernard, Abrami, Lou, Borokhovski, Wade, Wozney, Wallet, Fiset, and Huang 2004). Building on the contention that classroom and distance delivery could be equivalent, we were interested in a comparison of a remote teaching laboratory environment with the face-to-face environment. Our intent was to create a remote laboratory experience that is comparable with traditional laboratories. We wanted to determine whether facilitating real laboratories at a distance through remote technologies could be achieved and demonstrated within the context of specific experiments. In addition to our main consideration of sophisticated 
analytical instruments, we also briefly describe the remote control and monitoring of a simple physics experiment.

\section{Technical Details of Remote Access System Used}

A series of 15 different analytical instruments were interfaced to computers, which in turn were connected in a local area network (LAN) in a laboratory environment (see Figure 1). An Internet Security Accelerator Server (Reverse Publisher Server) was set-up to permit access to this LAN over a firewall using terminal emulation software. PC-Duo software was adopted for the terminal emulation to allow control of the desktop of a computer operating an instrument within that LAN. This arrangement was deployed so that the bulk of the software required to operate the instruments resides with the institution rather than on the remote workstation. The student or guest accessing any of the instruments requires only an Internet browser at their location.

Figure 1. Remote Access to Analytical Instruments

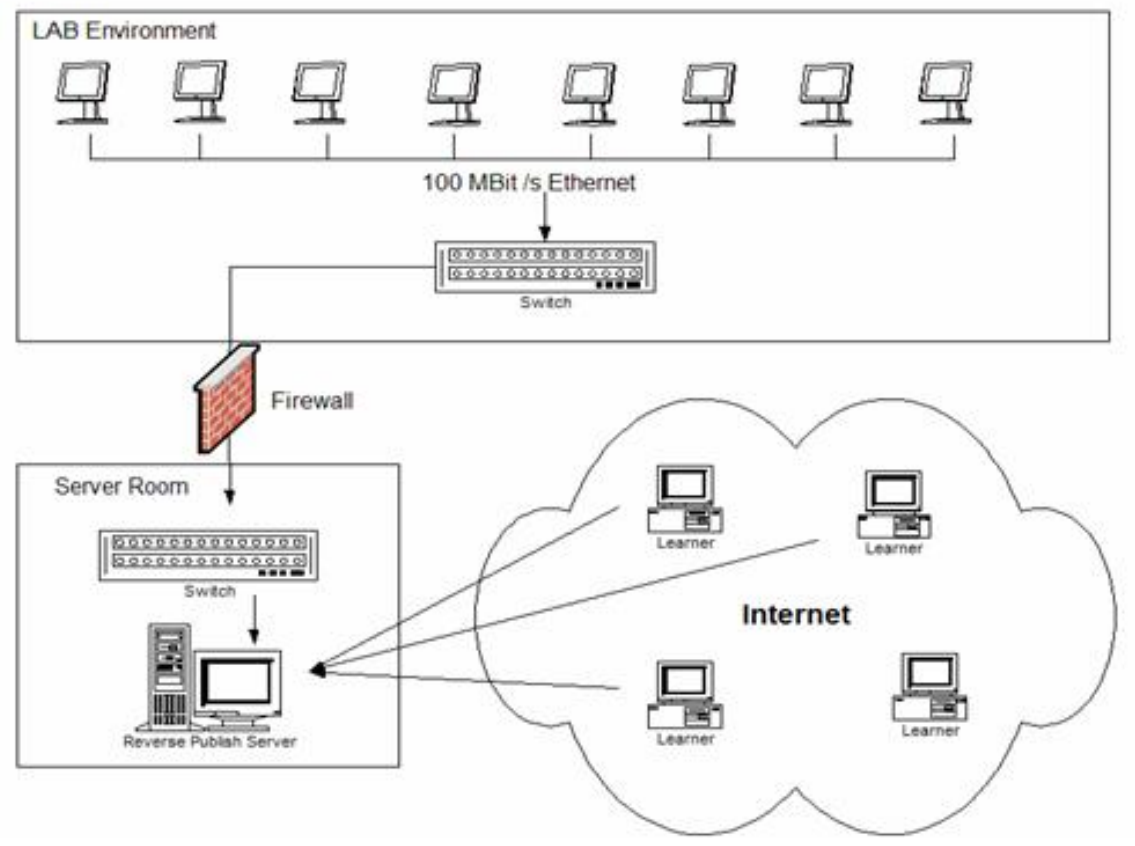

The following components were integrated into the website to facilitate access to performing a real time laboratory exercise and to provide a remote "screen" in the portal system, thus allowing students control of the analytical instrument: an Internet-based portal product, multimedia components, remote desktop control software, firewall security, self-contained webcams, streaming video server, NetSupport PC-Duo, TCP port assignment, scheduler, concurrent logons, and self-learning tutorials.

\section{Virtual versus Remote Access}

It is important here to differentiate between virtual and remote access. We define virtual access as interactive computer simulations of instrumentation and experiments. Virtual laboratories can prepare students for a real laboratory environment or conversely reinforce concepts from theory 
or experiment. In contrast, remote access allows students and/or researchers to physically carry out real experiments over the Web. Training involving Internet access to instruments and computers monitoring an experiment is a real laboratory experience. This experience far surpasses that which might be had using a simulation or computer program, and directly prepares students to use equipment commonly found in industrial or research laboratories. Students perform real-time analysis using equipment, methods, and skills that are common to modern analytical laboratories (or sophisticated teaching laboratories). Students obtain real results using real substances and make real conclusions, just as they would if they were in the laboratory with the equipment.

\section{Key Components of the Remote Lab Site}

It is not enough for students to have the ability to remotely connect with, and physically control, an analytical instrument to carry out an experiment. The site should be sufficiently self-contained so that novices can learn the basic chemical principles, as well as how to operate the instrument in a remote environment. This project was designed to build a seamless pedagogical front-end for instrument access in order to facilitate the high level of student learning and skills-development necessary to carry out an experiment at a distance. Some of the components that are incorporated into the Canadian Remote Sciences Laboratories (CRSC) website, found at www.remotelab.ca, include:

1. Public information describing the project, the researchers, and funding sources. This allows first-time visitors to get a brief glimpse of the site, the research being carried out, and to assure themselves of the credibility of the site and its developers.

2. Password protection to limit access to the tutorials and, more importantly, the actual instruments. This has the logistical function of addressing system security and prohibiting unauthorized use or potential vandalism.

3. FAQ and Help sections address common problems encountered by students. This is a proactive supply-side mediation approach designed to efficiently answer commonly anticipated questions.

4. Connection to the instructor for further address problems. Initially this was done asynchronously by email, but a chat feature on the website now provides more direct communication. This is a demand-side mediation approach designed to address specialized and real-time questions and needs of students. It is also intended to establish moral support for individual students through provision of a "teaching presence" connection (Anderson, Rourke, Archer, and Garrison, 2001).

5. Chemical principles tutorial to introduce students to the software, the instrument, and the particular experiment being undertaken. The purpose of the tutorial is to develop fundamental or remedial skills in the chemical principles, the instrumentation, and the online environment itself. General instrument tutorials consist of streaming videos that demonstrate use of instrumental software provided by the manufacturer to perform instrumental analysis. Interactive review exercises are also available to aid students in the recall of some of the key features of a particular aspect of the instrumental analysis software. Separate tutorials exist for particular experiments on a given instrument and may include downloadable text files. 
Figure 2. Streaming video demonstrating the instrument within the tutorial section

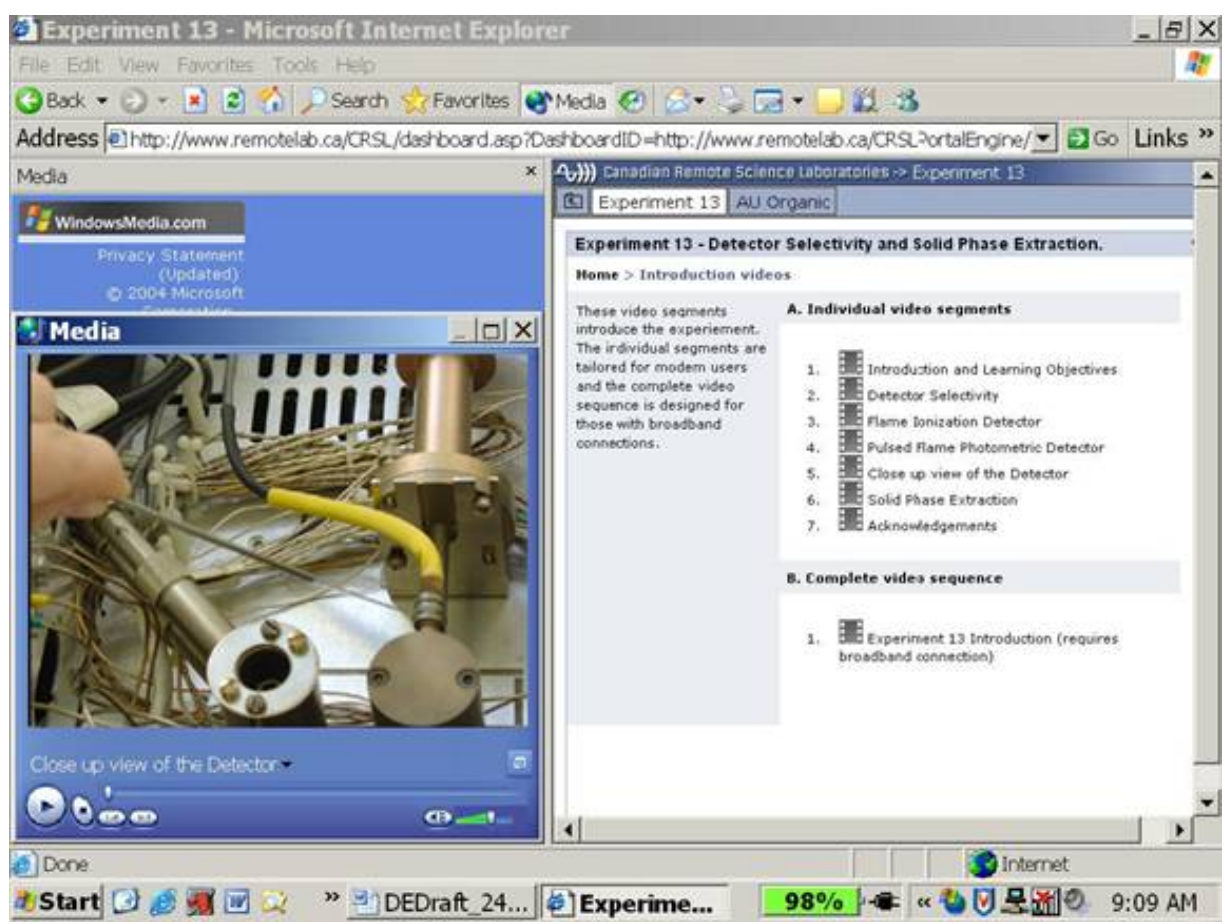

6. Qualifier exercises to establish a minimum skill level for students before moving onto actual instrument access. This provides scaffolding for students to help them master content and skills. It also limits exposing expensive instrumentation to complete novices, which may have the potential for frustration on all sides.

7. Scheduler function to help students and instructors assign unique and secure instrument time to qualified operators. This is a logistical function designed to insure effective use of these resources.

8. Actual instrument access to carry out experiments on real samples submitted earlier. This places students in a discovery or problem-based learning environment wherein they measure and collect real data. The intent is to reinforce upon students basic concepts and develop laboratory skills, while navigating potentially non-ideal results. 
Figure 3. Actual instrument access of chromatography experiment in progress with web camera view (insert) in real-time

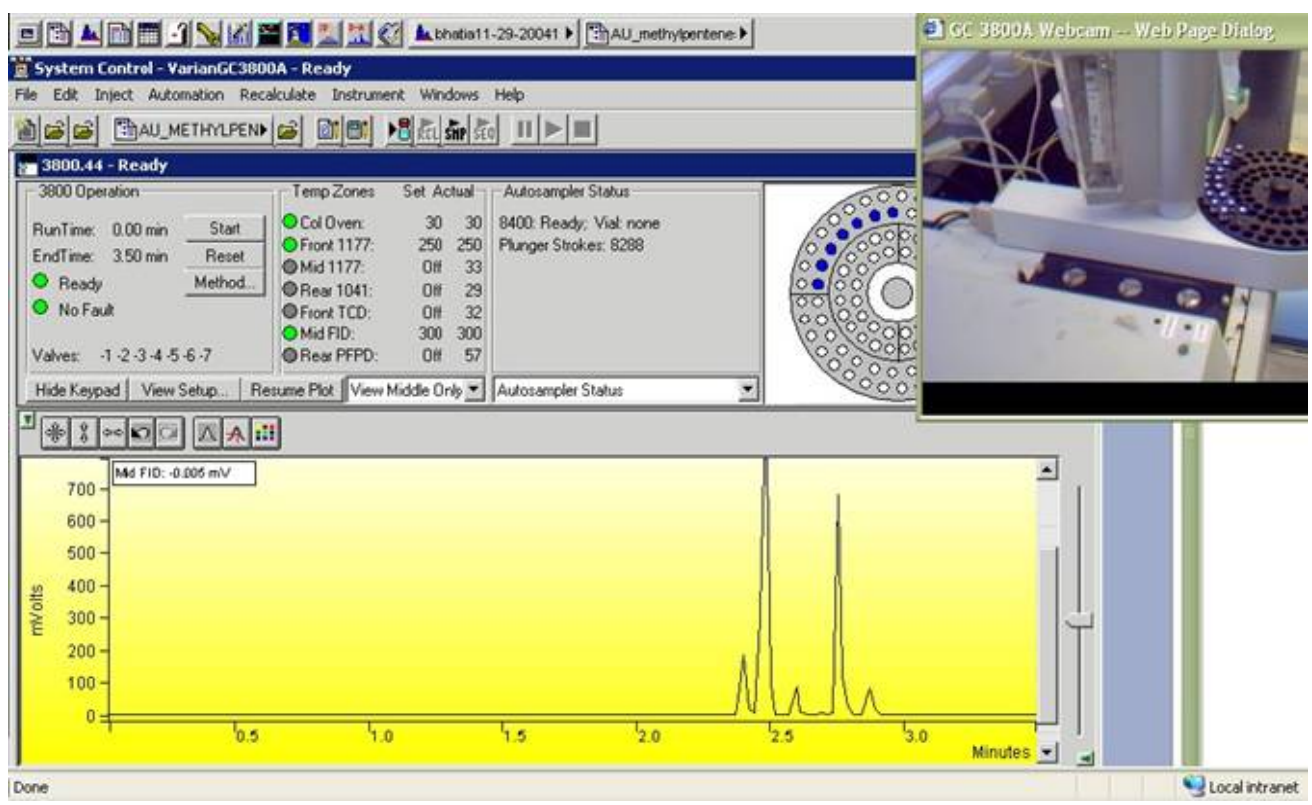

9. Web camera to allow real-time viewing of the instrument during the experiment. The purpose is to make the instrument real for the remote student - i.e., "seeing is believing."

10. Databases obtained mostly commercially with the instrument manufacturer and used as a reference library to compare with actual data obtained. This resource allows students to practice contrasting their measurements with more standardized or ideal results.

11. eLogbooks allow students to make comments and house data collected from the instrument. These "eLogbooks" are typically be submitted by students, either in whole or in part, for their laboratory report. The use of eLogbooks allow for the facile handling of large data sets collected, so students are not immersed in the details of preparing tables by hand, but instead focused on learning the actual experiment itself. It also provides students with a vehicle to develop their note taking skills, which is an important part of scientific experimentation.

12. Supplementary resource materials provided for each experiment guide learners to use additional information or useful links for further study. This is a gateway into related online literature rather than a comprehensive source. The purpose is as a resource for report preparation and to make students aware of the larger body of information available.

\section{Method}

The remote control environment for these chemistry experiments was new to both students and instructors. Indeed, many of the features incorporated were untested in the remote environment we created. Rather than construct rigorous measures to compare the remote and face-to-face environments in detail, we decided instead to examine student achievement and measure the attitude of individual students in the remote environment. Because we viewed this as a work in progress, this formative evaluation serves to provide initial feedback on an alternative laboratory delivery method, thus allowing us to use that information to direct future approaches. 
Both chromatography and spectroscopy are important techniques widely used in both research and industry. Chromatography is a technique that allows chemists to separate a mixture of compounds by running it through a chromatographic column. Each component has a characteristic separation rate, which can be used to identify it and therefore the composition of the original mixture. Spectroscopy is another useful analytical technique used to identify a substance by the characteristic spectrum of light (e.g., visible, ultraviolet, infrared) absorbed or emitted. Our remote instrumentation was modified for specific experiments in chromatography and spectroscopy within the regular teaching laboratory. Typically students attend a supervised faceto-face laboratory session where they prepare calibration standards and sample solutions on site. Once solutions have been prepared the vials containing the standards and samples are placed in the autosampler or autoinjector. The samples are then analyzed by the student on site or remotely. The onscreen workstation environment is identical for the operator whether he or she is working down the hall, off campus, or right beside the instrument itself. A login and password are assigned to students who have successfully completed the online tutorial and qualifier exercises; this allows them access and physical control of the instrument at pre-booked time slots. A group of 34 students carried out three experiments in an analytical course that involved remote access. They were surveyed on their experience at the end of each experiment, and the laboratory course grades were compared with students who did the same experiments face-to-face, in person.

\section{Varian and Agilent Chromatography Software (Experiment 1: Chromatography)}

During the first two weeks of the course, students worked through a series of tutorials designed to give them basic working knowledge of the software being used. Although called an experiment, no "wet chemistry" was carried out. Students were then surveyed on their experience.

\section{Fourier Transform Infrared (FT-IR) Spectroscopy Methods (Experiment 12: Spectroscopy)}

In this experiment students used a Nicolet Avatar 320 FT-IR instrument and viewed a video tutorial on the components and function of the FT-IR to examine the the effects of resolution and apodization functions on the quality of spectra; analyze a polymer using a horizontal attenuated total reflectance cell and observe the effects of scan number; perform a library search to identify the polymer; and perform quantitative analysis on ethyl acetate within a sample mixture following spectral subtraction during the laboratory component of this experiment. Before accessing the FT-IR remotely, students were required to examine an introductory tutorial that reviews the theoretical concepts behind the experiment and prepare the eLogbook by accessing the remotelab website. Students submitted their pre-lab eLogbook via email to their instructors before accessing the instrument; this precautionary step ensured solution preparation calculations and chemical safety aspects had been reviewed. Onsite time in the laboratory was required by students to prepare solutions and acquire spectra to be used when performing the experiments remotely. This required about one-half hour of onsite time before accessing the instrument. The time chosen by the students to perform this component of the experiment was flexible. After the lab was finished, students completed their eLogbook and submitted their report to their instructor via email. Students were then surveyed at the end of their course. 


\section{Detector Selectivity and Solid Phase Extraction (Experiment 13: Chromatography)}

In this experiment students compared the sensitivity and selectivity of two detectors on a Varian 3800A Gas Chromatograph: the flame ionization detector (FID) and a pulsed flame photometric detector (PFPD). Students also developed of a solid phase extraction (SPE) procedure for sample preparation. The wet chemistry, including the SPE procedure, was performed by students onsite in the laboratory. Samples were then placed in individual vials, which were then positioned on an autosampler of the Varian 3800A GC to be remotely accessed at a later time. Students were required to engage in a pre-lab exercise before the instrument was accessed. Once again, students were assigned to review an online text and/or audiovisual presentation using the remotelab website to examine the theoretical concepts for this experiment. Additionally, students had to prepare and submit their eLogbook via email prior to the experiment. An instrument .pdf file was a component of the website to enable instrument conditions to be documented within the eLogbook before entering the laboratory. Results and discussion were recorded by students in their eLogbook, which they submitted to their instructor by email. The chromatograms and reports once again had to be accessed from the laboratory printer. Students were then surveyed at the end of the course.

\section{Survey Results}

\section{Varian and Agilent Chromatography Software (Experiment 1: Chromatography)}

In this experiment 32 of the 34 students responded to the first survey, which was administered just prior to the laboratory examination two weeks into the term. All but four students reported using a highspeed connection (ADSL/ cable/ Ethernet) for their browser, and 31 of 32 students reported viewing the videos in the tutorial section. Students rated the quality of the tutorial videos on a five-point Likert scale. Students reported a high rate of satisfaction (4.3) on the size, clarity, sound, and smoothness of the videos. The students scored video clips slightly lower in the Understanding (4.0) and Appropriate Length (3.6) categories. This survey also had the students rate their preferred instructional format. The highest single method preferred by students was face-to-face or in-lab instruction (3.9); followed by online tutorials (3.5); and information given by text only (2.7). The choice of combining all three methods (4.3) was reported by students to be the most preferred instructional format, however.

\section{Fourier Transform Infrared (FT-IR) Spectroscopy Methods (Experiment 12: Spectroscopy)}

Only 15 of the 34 students responded to the survey. Although not necessary to operate the remote instrument, all but one student $(n=15)$ used high speed connections. Only nine students made use of the online tutorials, a much lower proportion than seen in Experiment 1 . The rest presumably relied on written instructions that could be downloaded or on prior knowledge. Quality ratings reported by students using a five-point Likert scale were similar, but slightly higher, when compared with Experiment 1 . That is, satisfaction with the size, clarity, sound, and smoothness of the videos (4.7); Understanding (4.1); and Appropriate Length (3.7). A summary of survey results of the tutorial videos is given in Table 1. It is interesting to note that the Appropriate Length scores did not correlate to the actual length of the video clips. That is, long 
videos were not necessarily more boring. For example, the "Discussion of the Library Search" (1:47 min) scored 3.5, while "Experiment Setup Window Entries" (5:15 min) scored 4.3. This seems to indicate that students' interest (and subsequent high/strong Length scores) could be maintained even though a video clip is longer. There were additional resource materials available on the website for student use; however, only one student made use of these materials. The ease of use of the eLogbook received an overall score of 4.7 by the students. There were some minor difficulties with the Solution Preparation and Example Calculations sections of the eLogbook for this experiment, but student comments indicate this might be more of a formatting problem.

An average rating of 2.8 was obtained for both ease of use of the instrument remotely versus onsite, as well as the overall rating of the remote access experience. There were comments about the technical aspects of the computer interface. Some students found the software to be slow, the screen display was not large enough for a few, and use of an instrument by more than one person at one time (students worked with partners) caused a little instrument control confusion. Two recurring comments offered by students about their experience in this experiment became apparent early on. First, students found the additional flexibility of doing tutorials, experiments, and searching databases from home to be valuable in this learning content. Second, students were constantly looking for in-person help.

Table 1. Selected Student Ratings ( $1=$ unacceptable to $5=$ high quality) of Online Tutorial Videos

Category

Size, Clarity, and Sound

Understanding

Appropriate Length
Experiment 1

4.3

4.0

3.6
Experiment 12

4.7

4.1

3.7
Experiment 13

4.0

4.5

4.0

\section{Detector Selectivity and Solid Phase Extraction (Experiment 13: Chromatography)}

Only 12 of the 34 students responded to this survey. Of those, 4 of the 12 students reported accessing the remote experiment using a modem connection, while the others reported using highspeed connections. Again, a larger proportion of students (11/12) viewed the videos in the tutorial portion of this experiment compared with Experiment 12. Quality ratings reported by students using a five-point Likert scale were significantly lower for videos. In this experiment, satisfaction with the size, clarity, sound, and smoothness of the videos (3.6 - 4.3) scored about a half point lower when compared to Experiment 1, which was conducted at the beginning of term. It also scored one full point lower than Experiment 12, even though the same format was used in all cases. Despite this, Understanding (4.5) and Length (4.0) obtained the highest scores. The eLogbook received an overall score of 4.8 by the students, which is almost identical to Experiment 12. This time the Solution Preparation and Example Calculations sections rated higher and were not seen as problem areas. Only two students reported using the additional resource materials available on the website.

Average ratings obtained for both ease of use of the instrument remotely versus onsite (3.3), as well as the overall rating of the remote access experience (3.2) were slightly up from the previous experiment (2.8). Again, similar comments on the technical aspects of the remote experience 
were received including screen display size. Also, the larger themes of desired in-person help and the convenience of the flexibility offered by remote access were also brought up by this group of students.

\section{Discussion}

The main focus of the Canadian Remote Sciences Laboratories website has been the chemistry experiments that involve student control of sophisticated analytical instruments. We also developed one simple physics experiment for first-year physics students. This remote lab experiment is used by students to determine the acceleration of gravity by dropping a steel ball. This device allows g (gravitational constant) to be determined to within 1 percent. Details of the experiment set-up and its evaluation will be described elsewhere. One of the more important features of this experiment was the use of a JVC web-cam, which allowed students to see the experiment in progress in real time. It is the combined feedback of both measured data values and images of the apparatus in motion that provide a true lab environment for the remote operator.

Remote control can be applied to both simple experiments and complex instruments. It therefore becomes crucial for educators to select an environment that is most appropriate for their students. A ball drop experiment seems to work well for first-year physics students, while the handling of complex analytical instruments works well for senior chemistry students. In a recent study, however, we had general first-year chemistry students remotely access an Ultraviolet-Visible Spectrophotometer to measure sample concentrations, which yielded mixed results (Baran, et al. 2004). Students reported that learning the instrument interface - whether on site or remotely was too much to ask for in a general first-year chemistry experiment, where only a handful of data points would be generated.

Nonetheless, the use of remote access offers distance educators in science another tool to provide a quality laboratory experience for their students. In our particular case in chemistry, remote control and the use of sophisticated analytical instruments are well-suited to each other. Since almost all current analytical instruments are already computer controlled, the ability to network them or set them up for remote access is relatively easy. In addition, exposure and practical experience with computer-controlled instruments - including remote access - is a valuable experience for students, as eventually they will be working with similar systems in industry. Other educators have also emphasized the importance of early introduction to modern instrumental methods at the undergraduate level in chemistry (Drew, 1996).

The mean laboratory course grade for students $(n=33)$ in the remote environment was 74.6 percent. The mean grades for two other cohorts $(n=46 ; n=47)$ not using the remote connection (in different years of the same course) were 76.4 and 78.6 percent respectively. A t-test was performed and the two groups in years where there was no online component resulted in a $\mathrm{P}$ value $(?=0.05)$ of 0.381 . A comparison of the online cohort $(74.6 \%)$ with each of the other non-online cohorts $(76.4$ and $78.6 \%)$ resulted in $P$ values $(?=0.05)$ of 0.491 and 0.117 , respectively. There are no significant differences in performance between groups of students carrying out analytical experiments on site versus remotely. So in terms of measuring by class grades, the learning achieved appears to be equivalent. From the students' perspective there has been a genuine appreciation for both the accessibility and the flexibility of remote access in the chemistry experiments we described. This was pronounced in our student surveys. As one student put it: "The single best aspect of the remote experience is that I could do the majority of the lab at home in my pajamas." Another student noted: "It can be accessed from anywhere, and simplifies the chromatography without pages and pages of chromatograms." That positive perspective also 
seems to be shared by students elsewhere involved with similar remote access studies reported in the literature. Students do not wish to be tied down in time or place to set laboratory exercise. The ability to work at one's own pace and at one's desired time, coupled with the ability to repeat experiments, is appealing. Remote access also has a potential advantage for students with disabilities. Not only does it address potential issues of safety, equipment handling, and physical access to the laboratory, the computer interface makes possible the use of suitable assistive technologies that might not be available in situ.

From an institution's perspective, remote experiments mean better use and sharing of equipment and less need to provide supervised laboratory sessions. It also opens the possibility of sharing expensive equipment for both teaching and research. From the instructors' perspective there have also been advantages. Online access means laboratory manuals can be readily upgraded, team teaching can be facilitated, and student work can be directly verified. In addition, the multiple user access to the website permits an instructor to observe students using instruments in the laboratory via remote access and to intervene using a chat tool when help is requested.

This brings us to the main criticism raised by students. Despite reporting a preference for a combination of instructional formats (text-based, online, and face to face), students also pronounced their desire to have in-person help when using the remote control access. Although students had access to tutorials, self tests, email, and the chat tool, they apparently preferred to speak face-to-face with a live instructor. This finding is also supported by the drop in participation rate in a group of 34 students from 32 in Experiment 1; to 15 in Experiment 12; and finally to 12 in Experiment 13. Why did this occur? In the first survey students prepared for the laboratory exam in the second week of the term where they needed to demonstrate proficiency in the use of the Varian and Agilent software. Instructors were not available to be consulted during that time. During the rest of the term laboratory instructors were available, however. Thus, there was no need for students to review the introductory tutorials related to the lab since the instructors were physically present to assist them understand the various features of the laboratory during the lab period.

Although students' desire for in-person help is apparent, the question we ask now is whether that need is real? Our experience teaching students in traditional supervised chemistry laboratories has been that there is an overwhelming tendency to ask the instructor questions first, rather than read the information in the laboratory manual. Could this be happening here too with the remote access laboratories? Are students seeking the path of least resistance? Or is it possible that the information presented on the website is not easily retrievable by students when they are trouble shooting. Perhaps there needs to be some sort of online help function designed to assist students retrieve the information they need? Another scenario to be considered is that students are simply looking for some level of human connection and reassurance.

Finally, we should make a brief comment on problem solving. Since these remote laboratories exist in the physical world with real experiments on real samples, there is also the possibility of operational problems, errors, and non-ideal results. In moderation, we view this as a beneficial for the student. Ironically, creators of some simulated experiments spend a lot of effort incorporating errors into their programs to make them more real and place the learner into a problem-solving environment. Our real-life experiments seem to do this automatically and we should see this as an opportunity to encourage learning.

We would like to summarize our experience and the discussion of our observations above as lessons learned. 
- Seeing is believing - using a camera to see the experiment is important to the student. (Although not investigated in our studies, this might eventually be extended to sound cues also.)

- It is important to match the experiment and student for the appropriate level of experimental and remote environment complexity. (Computer manipulations must reasonable for the eventual data obtained to seem worthwhile.)

- Students like the additional access and flexibility of the experiments. Faculty like making better use of existing instrumentation by employing them during non-business hours.

- Students reported great desire for in person instructor contact. However, is this needed or just wanted?

- Problems can be a good thing. Unexpected technical and experimental results in small quantities can lead to valuable learning opportunities.

\section{Future Directions}

This pilot study finds student performance to be equivalent for the remote laboratory experience and for face-to-face experiments. However, such scenarios should be explored in more detail so that the learning specific to the laboratory is more rigorously tested. With remote experiments incorporated into regular laboratory work, both the learning and the cost effectiveness of the approach will be easier to measure.

Our intent is to offer instruction and access to online experiments in a seamless package, one which is as self-contained as possible. As educators, we also want students to struggle and think about what they are doing, rather than being spoon-fed instant answers. On the other hand, any instruction should not be so inaccessible that students become lost or frustrated. In future modifications of the remote lab portal, we would like to specifically address the help function for the learner, while maintaining a self-contained online package for both teaching and instrument access. We also feel that it would be informative to pilot some of these experiments with groups of students that are well removed from the physical location of the analytical instruments and the instructors. This would effectively require students to communicate and learn on their own at a distance, rather than dropping by an instructor's office down the hall for in-person help. Another feature we would like to incorporate is a trouble-shooting flowchart, or decision tree, or autonomous agent, to assist student learning online. Such a feature would be in addition to FAQ pages that assist students with commonly encountered technical problems or misconceptions. We would also like to develop a video connection to facilitate more intimate communication between instructors and students while maintaining a remote environment. We have already discovered in both the physics and chemistry experiments that a visual image is powerful. It is possible for students to control an instrument using only the on-screen display offered by the instrument manufacturer. If, however, they see the instrument moving in real time as they are controlling it, the experience is enhanced. They feel it is real.

We have implemented and tested a complete remote laboratory environment for chemistry and tried out one physics online experiment. There are numerous advantages to remote experimentation and it is a natural adjunct to courses in which the other components are also offered by distance education. Since at least in upper level chemistry, remote control techniques 
for instruments closely parallel those used in an onsite laboratory itself, this is a natural subject area for further implementation of the remote environment we have developed. With good feedback and ancillary materials we suggest that distance education implementation of laboratories through remote access techniques can only gain in importance and contribute in a significant way to science education.

\section{Acknowledgements}

We appreciate Athabasca University project students Edna Cabalo, Jason Ponto, and Christy Tkatchuk who contributed to several key areas of the project development. We also thank Randy Troppman (NAIT) for assistance with areas of application development and web site design.

We are grateful for financial support of our home institutions NAIT and Athabasca University and funding through the Office of Learning Technology, a department of Human Resources Development Canada.

\section{References}

Anderson, T., Rourke, L., Archer, W., and Garrison, R. (2001). Assessing teaching presence in computer conferencing transcripts. Journal of the Asynchronous Learning Network, 5(2) Retrieved September 17, 2005, from: http://www.aln.org/alnweb/journal/jalnvol5issue2v2.htm

Baran, J., Currie, R., and Kennepohl, D. (2004). Remote Instrumentation for the Teaching Laboratory. Journal of Chemical Education, 81(12), 1814 - 1816.

Bernard, R. M., Abrami, P.C., Lou, Y., Borokhovski, E., Wade, A., Wozney, L., et al. (2004). How Does Distance Education Compare With Classroom Instruction? A Meta-Analysis of the Empirical Literature. Review of Educational Research, 74(3), 379 - 439.

Cooper, M. (2000). PEARL: Effective learning through remote experiments. A collection of papers by various authors. Retrieved September 26, 2005 from: http://iet.open.ac.uk/pearl/index.htm

Connors, M. (2004). A Decade of Success in Physics Distance Education at Athabasca University. Physics in Canada, 60(1), 49 - 54.

Drew, S. M. (1996). Integration of National Instruments' LabVIEW Software into the Chemistry Curriculum. Journal of Chemical Education, 73(12), 1107 - 1111.

Gostowski, R. (1996). Teaching Analytical Instrument Design with LabVIEW. Journal of Chemical Education, 73(12), 1103 - 1106.

Haines, R. S. (1998). Teaching Computer Concepts to Undergraduate Chemists. Journal of Chemical Education, 75(6), 785 - 787.

Hesselink, L., Rizal, D., and Bjornson, E. (2000). CyberLab: Remote access to laboratories through the world-wide-web. Retrieved September 26, 2005 from: http://www.discoverlab.com/References/043.pdf 
Holmberg, R. G., and Bakshi, T. S. (1992). Postmortem on distance education course: Successes and failures. American Journal of Distance Education, 6(1) 27 - 39.

Kennepohl, D., and Last, A. (1997). Science at a Distance. Journal of College Science Teaching, 27(1), $35-38$.

Kennepohl, D., and Last, A. (2000). Teaching Chemistry at Canada’s Open University. Distance Education, 21(1), 183 - 197.

Kennepohl, D. (2001). Using Computer Simulations to Supplement Teaching Laboratories in Chemistry for Distance Delivery,. Journal of Distance Education., 16(2), 58 - 65.

Spanoghe, P., Cocquyt, J., and Van der Meeren, P. (2001). A Low-Cost Dynamic Surface Tension Meter with a LabVIEW Interface and Its Usefulness in Understanding Foam Formation. Journal of Chemical Education, 78(3), 338 - 342.

Zurn, A., Paasch, S., Thiele, S., and Salzer, R. (2003). Linked Curriculum Chemistry: Different from a virtual university, CHIMIA, 57(3), $105-115$.

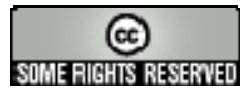

